\title{
Umbilical Region
}

National Cancer Institute

\section{Source}

National Cancer Institute. Umbilical Region. NCI Thesaurus. Code C33829.

The central area of the abdomen that surrounds the umbilicus; it lies inferior to the epigastric region, superior to the suprapubic region, and is bounded laterally by the two lumbar regions. 